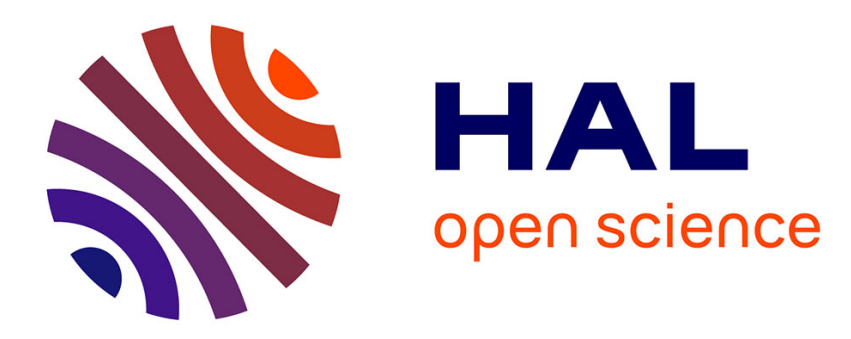

\title{
The coupling of multi-phase flow models Jean-Marc Hérard
}

\section{To cite this version:}

Jean-Marc Hérard. The coupling of multi-phase flow models. 42nd AIAA Fluid Dynamics Conference and Exhibit, Jun 2012, New Orleans, United States. 10.2514/6.2012-3357 . hal-01582643

\section{HAL Id: hal-01582643 \\ https://hal.science/hal-01582643}

Submitted on 5 Apr 2018

HAL is a multi-disciplinary open access archive for the deposit and dissemination of scientific research documents, whether they are published or not. The documents may come from teaching and research institutions in France or abroad, or from public or private research centers.
L'archive ouverte pluridisciplinaire HAL, est destinée au dépôt et à la diffusion de documents scientifiques de niveau recherche, publiés ou non, émanant des établissements d'enseignement et de recherche français ou étrangers, des laboratoires publics ou privés. 


\title{
The Coupling of Multi-Phase Flow Models
}

\author{
Jean-Marc Hérard* \\ EDF, RED, 6 quai Watier, 78400, Chatou, France.
}

\begin{abstract}
This paper provides an overview of some recent developments that were motivated by the unsteady interfacial coupling of existing codes. We describe the framework and the basic ideas first, which mainly rely on the use of a father model. Next we list the main achievements in this domain, and focus then on the interfacial coupling of a three-field model with a two-fluid two-phase flow model. Some numerical results are shown, and we eventually try to point out the main results and give some perspectives for further work.
\end{abstract}

\section{Introduction}

This paper presents a partial review of some recent work that has been achieved in the NEPTUNE project since $2002,{ }^{22}$ which concerns the unsteady interfacial coupling of distinct codes, or equivalently of distinct models devoted to the simulation of water-vapour two-phase flows.

One of the main motivations for such a work was tightly linked with the fact that many distinct two-phase flow codes have been built during the last decades, and that users ask for coupling procedures that enable to perform stable and meaningful coupled simulations with different codes. In the French nuclear safety framework, these codes include THYC, FLICA, CATHARE and NEPTUNE_CFD codes. ${ }^{22}$ The first two rely on the homogeneous approach, whereas the third one and the fourth one use the two-fluid approach as a main guideline. Moreover, the CATHARE code also allows to compute solutions of a three-field model $\left({ }^{34}\right)$ in some specific situations; a straightforward consequence is that there is a need for the coupling of a three-field model with a two-fluid model. All of these codes compute approximations of solutions of models with the Finite Volume method, using either the staggered approach (THYC, CATHARE) or the colocated approach (FLICA, NEPTUNE_CFD). The main objective of the present paper is to present the main principles that have been considered in order to construct coupling procedures and to give a brief review of achievements. We will also give some focus on the recent interfacial coupling of a two-fluid model with a three-field model, and eventually we will discuss some possible advantages and drawbacks of our approach.

In practice, we will need to define two sudomains $\Omega_{l}=\{x<0\}$ and $\Omega_{r}=\{x>0\}$, and a coupling interface standing at $x=0$. Thus we will focus on models of the form:

$$
\partial_{t}\left(W_{l}\right)+\partial_{x}\left(\left(f_{l}\left(W_{l}\right)\right)\right)+C_{l}\left(W_{l}\right) \partial_{x}\left(\left(g_{l}\left(W_{l}\right)\right)\right)=S_{l}\left(W_{l}\right) \quad \text { for: } \quad x<0,
$$

and:

$$
\partial_{t}\left(W_{r}\right)+\partial_{x}\left(\left(f_{r}\left(W_{r}\right)\right)\right)+C_{r}\left(W_{r}\right) \partial_{x}\left(\left(g_{r}\left(W_{r}\right)\right)\right)=S_{r}\left(W_{r}\right) \quad \text { for: } \quad x>0,
$$

where the state variables $W_{l}$ and $W_{r}$ are distinct and lie in $\mathcal{R}^{p}$ and $\mathcal{R}^{q}$ respectively. In most cases $p$ and $q$ are distinct, and the main problem is to provide meaningful boundary conditions at each time step through the coupling interface. The left hand sides of the latter models gather the convective effects, and may contain non conservative contributions (in that case $C_{l}$ an $C_{r}$ are non zero). The right hand sides (so called source terms) contain zero components and non-zero contributions that involve relaxation time scales.

${ }^{*}$ Senior engineer, EDF, R\&D, Fluid Dynamics, Power Generation and Environment, 6 quai Watier, 78400, Chatou, France. 


\section{An interfacial coupling approach based on the father model}

\section{A. Basic ideas}

The basic idea of our approach relies on the use of a father model in order to derive suitable boundary conditions at $x=0^{+}$and $x=0^{-}$. The underlying idea may in fact be found in the early paper by Greenberg and Leroux ${ }^{21}$ though not formalized exactly as it is done below. Actually, once the father model has been chosen, the procedure is quite simple. The sequence is as follows:

- Step 1: Define the father model (1) at the coupling interface;

- Step 2: Define and reconstruct interface conditions IC on both sides of the interface $x=0$ at each time step;

- Step 3: Solve the exact (or approximate) Riemann problem corresponding to the father model (1), using these IC;

- Step 4: Plug the interface values in a numerical flux using both states $W_{F}\left(0^{-}\right)$and $W_{F}\left(0^{+}\right)$and update cell values with the Finite Volume scheme, at each time step.

Remark 1:

Of course, steps 3 and 4 are classical and thus are not discussed herein. However, in many practical cases, the exact solution of the Riemann problem is not available ; hence approximate Riemann solvers are used in such a situation.

Remark 2:

The father model may contain a steady wave (this may happen for instance when coupling a fluid flow model and a porous model) ; in that case the interface values $W_{F}\left(0^{-}\right)$and $W_{F}\left(0^{+}\right)$may be distinct. This will be detailed when discussing the coupling problem.

\section{B. Constraints on the father model}

The governing equations of the father model are:

$$
\partial\left(W^{F}\right)+\partial\left(H^{F}\left(W^{F}\right)\right)+D^{F}\left(W^{F}\right) \partial_{x}\left(W^{F}\right)=S\left(W^{F}\right)
$$

where $W^{F}$ lies in $\mathcal{R}^{s}$, where $\max (p, q) \leq s$. The father model should enjoy the following properties:

- C1: The left-hand side of (1) is an hyperbolic problem (where the resonance phenomenon may appear);

- C2: Smooth solutions of (1) must comply with an entropy inequality;

- C3: Jump conditions associated with (1) must be unique;

- C4: Expected positive quantities should be preserved by (1);

- C5: System (1) should contain the inner mechanisms such that left and right models can be retrieved through relaxation processes (or at least through the use of adequate colour functions).

Remark 3:

These constraints deserve at least a few comments. The first four conditions (C1, C2, C3, C4) are obviously compulsory in order to provide a meaningful solution of the Riemann problem at the coupling interface, which is of course the keystone of the approach. Moreover, the last condition (C5) will give the links that guarantee a meaningful coupling procedure. Of course, these constraints will not be sufficient in order to provide a unique candidate for a given coupling problem, which may be viewed as an a priori drawback.

\section{Reconstruction of interface initial conditions}

The reconstruction is mandatory when $p<s$ ( or $q<s$ ). The exact procedure depends on the coupling problem. We will discuss that point later on, when giving more details on the father model, for a given example. 


\section{A brief review of some interfacial coupling experiments}

Various coupling problems have been investigated, and we will only consider here a few among them. Almost all of them concern the coupling of multiphase flow models, but at least one is different, which refers to the coupling of a 1D model with the same model in a two-dimensional framework. This is an important problem since many 1D codes have been built in order to simulate flows in pipes and other components where the flow is assumed to be strictly 1D. We refer the reader to the paper, ${ }^{28}$ which investigates this problem in detail. In that particular case, two distinct father models were proposed. The first one was a non-conservative model, whereas the second one was in conservation form. It has been shown in $^{28}$ that the first one should be prefered, for stability reasons, by comparing the full two-dimensional computations with the coupled simulations. It must be noted that the validation of the coupling approach is rather easy in that case, since the reference solution -that is to say the full $2 \mathrm{D}$ simulation- is obvious and unique.

The following coupling problems have been investigated :

- E1: The interfacial coupling of an homogeneous equilibrium model $(p=3)$ with an homogeneous relaxation model $(q=4)$;

- E2: The interfacial coupling of a two-fluid hyperbolic model $(p=7)$ with an homogeneous relaxation model $(q=4)$;

- E3: The interfacial coupling of a classical two-fluid model $(p=6)$ with an homogeneous relaxation model $(q=4)$;

- E4: The interfacial coupling of a two-fluid porous model $(p=8)$ with a two-fluid fluid model $(q=7)$ (or its counterpart with the homogeneous approach $-p=4$ and $q=3$-);

- E5: The interfacial coupling of a two-fluid hyperbolic model $(p=7)$ with a three-field model $(q=11)$.

The reader may find details on the coupling problem (E1) in references. ${ }^{2,5}$ The father model used in the second reference is an homogeneous relaxation model that includes a colour function (thus $s=5$ ) which enables to select the relevant equation of state on each subdomain $\Omega_{l, r}$. References ${ }^{29}$ and $^{27}$ focus on the coupling of problems (E2) and (E3), which are indeed quite similar. The father model in both cases corresponds to the hyperbolic two-fluid model (2) introduced in the next section $(s=7)$. This model enables to retrieve the standard two-fluid model, ${ }^{31}$ assuming that the pressure relaxation time scale $\tau_{P}$ is set to zero. A similar remark holds for the homogeneous relaxation model, while enforcing $\tau_{T}=\tau_{U}=\tau_{P}=0$, where $\tau_{U}$ and $\tau_{T}$ denote the velocity and temperature relaxation scales. The father model involved in the coupling problems belonging to class (E4) corresponds to the porous model, when focusing on the homogeneous approach $^{23,24}$ or on the two-fluid approach. ${ }^{15,17,26}$ Details on reconstruction procedures can be found in the above-mentionned references. These reconstructions may become difficult when using complex equations of state ; in fact they usually require solving non-linear scalar equations in cells touching the coupling interface. The last coupling problem (E5) that we briefly present below was mainly motivated by some developments in the CATHARE code $\left(\operatorname{see}^{16,22,34}\right)$.

\section{The interfacial coupling of a three-field model with a two-phase flow model}

We only give in this extended abstract the basic models that must be coupled through the interface. Details on the two-fluid model can be found in ${ }^{12,14}$ and related references ( $\operatorname{see}^{7,8,32}$ ). The three-field model is examined $\mathrm{in}^{25}$; it corresponds to the model ${ }^{22,34}$ when an instantaneous return to pressure equilibrium is assumed. Both models comply with constraints (C1-C4). We present both models in the following two subsections, and then show a few numerical results of unsteady coupled simulations. Many details and numerical results can be found in. ${ }^{16}$

\section{A. Governing equations of the two-fluid model}

We use the following notations: the liquid and vapour phases are indexed by $\mathbf{l}$ and $\mathbf{v}$ respectively. Governing equations of the two-fluid model may be written: 


$$
\left\{\begin{array}{l}
\partial_{t}\left(\alpha_{l}\right)+V_{I} \partial_{x}\left(\alpha_{l}\right)=\phi_{l} \\
\partial_{t}\left(m_{l}\right)+\partial_{x}\left(m_{l} U_{l}\right)=0 \\
\partial_{t}\left(m_{v}\right)+\partial_{x}\left(m_{v} U_{v}\right)=0 \\
\partial_{t}\left(m_{l} U_{l}\right)+\partial_{x}\left(m_{l} U_{l}^{2}\right)+\alpha_{l} \partial_{x}\left(P_{l}\right)+\left(P_{l}-P_{I}\right) \partial_{x}\left(\alpha_{l}\right)=S_{l} \\
\partial_{t}\left(m_{v} U_{v}\right)+\partial_{x}\left(m_{v} U_{v}^{2}\right)+\alpha_{v} \partial_{x}\left(P_{v}\right)+\left(P_{v}-P_{I}\right) \partial_{x}\left(\alpha_{v}\right)=-S_{l} \\
\partial_{t}\left(\alpha_{l} E_{l}\right)+\partial_{x}\left(\alpha_{l} U_{l}\left(E_{l}+P_{l}\right)\right)+P_{I} \partial_{t}\left(\alpha_{l}\right)=V_{I} S_{l}+\psi_{l} \\
\partial_{t}\left(\alpha_{v} E_{v}\right)+\partial_{x}\left(\alpha_{v} U_{v}\left(E_{v}+P_{v}\right)\right)+P_{I} \partial_{t}\left(\alpha_{v}\right)=-V_{I} S_{l}-\psi_{l}
\end{array}\right.
$$

where $\alpha_{l}$ and $\alpha_{v}$ denote the liquid and vapour statistical void fractions, assuming that $\alpha_{l}>0$ and $\alpha_{v}>0$ are such that:

$$
\alpha_{l}+\alpha_{v}=1
$$

$U_{l}, P_{l}, \rho_{l}, m_{l}=\alpha_{l} \rho_{l}$ denote the velocity, pressure, density, partial mass of the liquid phase (respectively $U_{v}$, $P_{v}, \rho_{v}, m_{v}=\alpha_{v} \rho_{v}$ for the vapour phase). Total energies within each phase are :

$$
E_{l, v}=\frac{1}{2} \rho_{l, v}\left(U_{l, v}\right)^{2}+\rho_{l, v} e_{l, v}\left(P_{l, v}, \rho_{l, v}\right)
$$

where $e_{l, v}$ stands for the internal energy function. We need to prescribe closure laws, which comply with the entropy inequality. These read:

$$
\begin{aligned}
& \phi_{l}=\alpha_{v} \alpha_{l}\left(P_{l}-P_{v}\right) / \tau^{P} / \Pi_{0} \\
& S_{l}=\frac{m_{l} m_{v}}{m_{l}+m_{v}}\left(U_{v}-U_{l}\right) / \tau^{U} \\
& \psi_{l}=\left(C_{v}\right)_{0} \frac{m_{l} m_{v}}{m_{l}+m_{v}}\left(T_{v}-T_{l}\right) / \tau^{T}
\end{aligned}
$$

where $\tau^{P} \geq 0, \tau^{U} \geq 0$ and $\tau^{T} \geq 0$ are relaxation time scales that govern return to equilibrium for pressures, velocities and temperatures. The couple $\left(P_{I}, V_{I}\right)$ is set to $\left(P_{v}, V_{l}\right)\left(\mathrm{see}^{7,8}\right)$.

\section{B. Governing equations of the three-field model}

This model $\left({ }^{34}\right)$ is assumed to be suitable to represent flows containing liquid droplets (indexed by $l d$ ) that should not be confused with a continuous liquid phase (indexed by $l c$ ), and a vapour phase (indexed by $v$ ). We use the same notations as in the previous subsection for phase fractions, densities, velocities, pressures and temperatures within each phase. Phase fractions must agree with the local rule $\left(\alpha_{l c}+\alpha_{l d}+\alpha_{v}\right)(x, t)=1$. We omit in this paper the mass transfer in order to simplify the whole presentation. Thus, the governing equations of the three-field model are:

$$
\left\{\begin{array}{l}
\partial_{t}\left(\alpha_{l c}\right)+U_{l d} \partial_{x}\left(\alpha_{l c}\right)=\phi_{l c} \\
\partial_{t}\left(\alpha_{v}\right)+U_{l d} \partial_{x}\left(\alpha_{v}\right)=\phi_{v} \\
\partial_{t}\left(m_{l c}\right)+\partial_{x}\left(m_{l c} U_{l c}\right)=0 \\
\partial_{t}\left(m_{v}\right)+\partial_{x}\left(m_{v} U_{v}\right)=0 \\
\partial_{t}\left(m_{l d}\right)+\partial_{x}\left(m_{l d} U_{l d}\right)=0 \\
\partial_{t}\left(m_{l c} U_{l c}\right)+\partial_{x}\left(m_{l c} U_{l c}^{2}\right)+\alpha_{l c} \partial_{x}\left(P_{l c}\right)=S_{l c} \\
\partial_{t}\left(m_{v} U_{v}\right)+\partial_{x}\left(m_{v} U_{v}^{2}\right)+\alpha_{v} \partial_{x}\left(P_{v}\right)=S_{v} \\
\partial_{t}\left(m_{l d} U_{l d}\right)+\partial_{x}\left(m_{l d} U_{l d}^{2}\right)+\alpha_{l d} \partial_{x}\left(P_{l d}\right)+\left(P_{l c}-P_{l d}\right) \partial_{x}\left(\alpha_{l c}\right)+\left(P_{v}-P_{l d}\right) \partial_{x}\left(\alpha_{v}\right)=-S_{l c}-S_{v} \\
\partial_{t}\left(\alpha_{l c} E_{l c}\right)+\partial_{x}\left(\alpha_{l c} U_{l c}\left(E_{l c}+P_{l c}\right)\right)+P_{l c} \partial_{t}\left(\alpha_{l c}\right)=U_{l d} S_{l c}+\psi_{l c} \\
\partial_{t}\left(\alpha_{v} E_{v}\right)+\partial_{x}\left(\alpha_{v} U_{v}\left(E_{v}+P_{v}\right)\right)+P_{v} \partial_{t}\left(\alpha_{v}\right)=U_{l d} S_{v}+\psi_{v} \\
\partial_{t}\left(\alpha_{l d} E_{l d}\right)+\partial_{x}\left(\alpha_{l d} U_{l d}\left(E_{l d}+P_{l d}\right)\right)-P_{l c} \partial_{t}\left(\alpha_{l c}\right)-P_{v} \partial_{t}\left(\alpha_{v}\right)=-U_{l d} S_{l c}-U_{l d} S_{v}-\psi_{l c}-\psi_{v}
\end{array}\right.
$$

Internal energy functions are the same for liquid droplets and for the continuous liquid phase :

$$
e_{l c}(P, \rho)=e_{l d}(P, \rho) \stackrel{\text { def }}{=} e_{l}(P, \rho)
$$

Source terms corresponding to drag effects, heat transfer, and return to pressure equlibrium, are :

$$
\begin{aligned}
& \phi_{l c}=\alpha_{l c} \alpha_{l d}\left(P_{l c}-P_{l d}\right) / \tau_{l c}^{P} / \Pi_{0} \\
& \phi_{v}=\alpha_{v} \alpha_{l d}\left(P_{v}-P_{l d}\right) / \tau_{v}^{P} / \Pi_{0}
\end{aligned}
$$




$$
\begin{gathered}
S_{l c}=\frac{m_{l c} m_{l d}}{m_{l c}+m_{l d}}\left(U_{l d}-U_{l c}\right) / \tau_{l c}^{U} \\
S_{v}=\frac{m_{v} m_{l d}}{m_{v}+m_{l d}}\left(U_{l d}-U_{v}\right) / \tau_{v}^{U} \\
\psi_{l c}=C_{v}\left(\frac{m_{l c} m_{l d}}{m_{l c}+m_{l d}}\right)\left(T_{l d}-T_{l c}\right) / \tau_{l c}^{T} \\
\psi_{v}=C_{v}\left(\frac{m_{v} m_{l d}}{m_{v}+m_{l d}}\right)\left(T_{l d}-T_{v}\right) / \tau_{l c}^{T}
\end{gathered}
$$

Again $\tau_{k}^{P} \geq 0, \tau_{k}^{U} \geq 0$, and $\tau_{k}^{T} \geq 0$ denote relaxation time scales for pressure, velocity and temperature. The counterpart of liquid variables in the two-fluid model (2) are:

$$
\begin{aligned}
& \alpha_{l} \stackrel{\text { def }}{=} \alpha_{l c}+\alpha_{l d} \\
& m_{l} \stackrel{\text { def }}{=} \alpha_{l c} \rho_{l c}+\alpha_{l d} \rho_{l d} \\
& m_{l} U_{l} \stackrel{\text { def }}{=} m_{l c} U_{l c}+m_{l d} U_{l d} \\
& \alpha_{l} E_{l} \stackrel{\text { def }}{=} \alpha_{l c} E_{l c}+\alpha_{l d} E_{l d}
\end{aligned}
$$

\section{The father model and the reconstruction procedure}

The father model that is chosen in the coupling procedure is the three-field model. This choice means that the reconstruction procedure must only be applied on the side corresponding to the two-fluid model, so that:

$$
\begin{aligned}
& T_{l c}=T_{l d} \stackrel{\text { def }}{=} T_{l} \\
& U_{l c}=U_{l d} \stackrel{\text { def }}{=} U_{l} \\
& P_{l c}=P_{l d} \stackrel{\text { def }}{=} P_{l}
\end{aligned}
$$

\section{A few numerical experiments}

We show below two different coupled simulations.

1. In the first one, a Riemann problem generates waves in the three-field region (for $x<0.525$ ). The initial discontinuity is located at $x=0.5$ and the right-going waves propagate and hit the coupling interface $x=0.525$ after a while. We may examine in figure 1 the behaviour of the void fraction profiles of the liquid droplet field $\alpha_{l d}$, considering two different meshes. The coarse grid (on the left side) contains one thousand regular cells and the fine one (on the right side) contains 50000 cells. We notice a small glitch around the coupling interface on the finer mesh that vanishes when time increases (see figure 1).
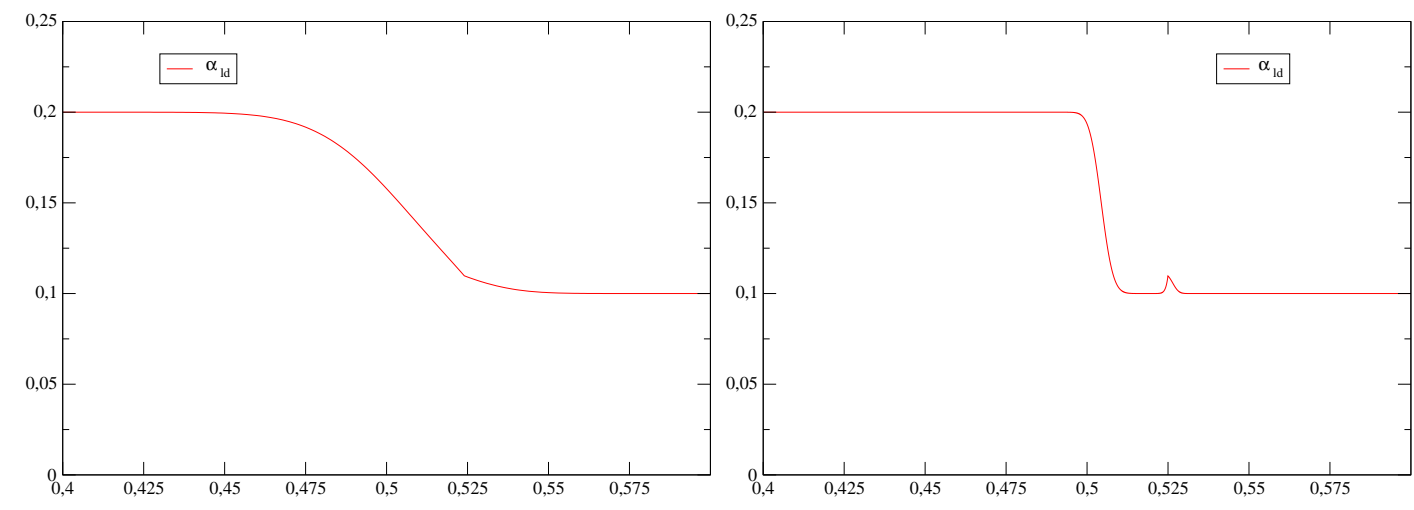

Figure 1. Test 1: liquid droplet void fraction profile. Coarse mesh (left), fine mesh (right).

2. The second coupled simulation is similar, but the Riemann problem occurs now in the two-fluid region $(x=0.5)$. Left-going waves hit the coupling interface $x=0.475$ some time later. Results are again displayed for two mesh refinements (see figure 2). Obviously the behaviour of the coupled simulation is again stable ; moreover, one can hardly observe any perturbation around the coupling interface now. This was expected actually, since the relaxation process no longer constrains the flow, unlike in the previous case. 

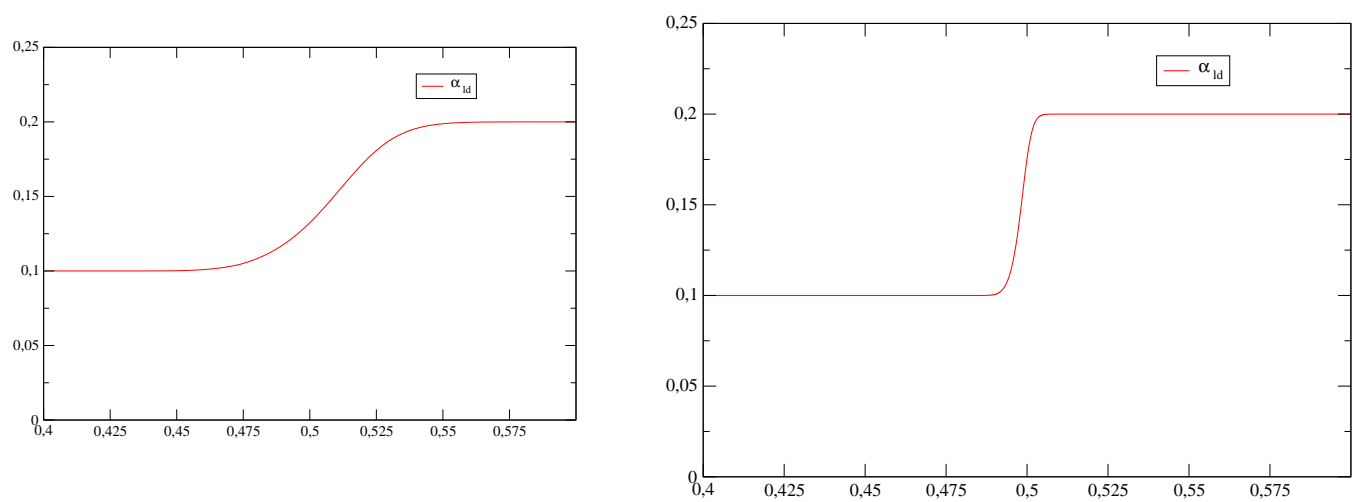

Figure 2. Test 2: liquid droplet void fraction profile. Coarse mesh (left), fine mesh (right).

\section{Conclusion}

Techniques that have been presented and discussed herein provide rather simple and stable tools in order to perform coupled simulations of distinct codes in the framework of fluid flow models. The present approach that relies on the father model benefits from several advantages:

- It may be used in Finite Volume codes or other codes (for instance codes based on FE methods) since it provides interface states on each side of the coupling interface ;

- It may be used in Finite Volume codes relying on the staggered appproach or the colocated approach ;

- It does not require the computation of the exact interface Riemann solution ;

- It enables -and requires- to get a deep knowledge of models to be coupled and in particular it gives focus on the possible hierarchy between two-phase flow models through relaxation process.

Nonetheless, we point out the fact that well-balanced schemes are sometimes mandatory at the coupling interface, in particular when the father model contains some steady wave ; otherwise schemes may generate stable approximations that converge towards wrong solutions ( $\mathrm{see}^{15}$ and also ${ }^{33}$ for instance). Another difficulty that should not be hidden concerns the verification of the coupled simulations. For a few coupling procedures, exact solutions may be exhibited ; however, some coupling problems can only be verified in asymptotic situations.

Eventually, we would like to emphasize that some other ideas to tackle the problem of the interfacial coupling of fluid models have been introduced and detailed in a recent series of papers $\left(\operatorname{see}^{2-4,9-11,13,18-20}\right)$ by members of the working group. ${ }^{1}$

\section{Acknowledgments:}

Part of this work has been achieved with PhD students Laetitia Girault and Olivier Hurisse, who received financial support by EDF through EDF/CIFRE contracts. All computational facilities were provided by EDF. It has also benefited from fruitful discussions with members of the working group [1]: Annalisa Ambroso, Christophe Chalons, Frédéric Coquel, Edwige Godlewski, Frédéric Lagoutière, Pierre-Arnaud Raviart, and Nicolas Seguin, and also with Thierry Gallouët. The main part of this work has been achieved in the framework of the NEPTUNE project, with financial support by CEA (Commissariat à l'Energie Atomique et aux Energies Alternatives), EDF, IRSN (Institut de Radioprotection et de Sureté Nucléaire) and AREVA-NP. 


\section{References}

1 Ambroso, A., Chalons, C., Coquel, F., Godlewski, E., Lagoutiere, F., Raviart, P.A., And Seguin, N., "Working group on the interfacial coupling of fluid models" http://www.ann.jussieu.fr/groupes/cea/, 2003.

2 Ambroso, A., Chalons, C., Coquel, F., and Godlewski, E., Lagoutiere F., Raviart P.A., Seguin N., "The coupling of homogeneous models for two-phase flows", International Journal of Finite Volumes, URL: http://www.latp.univ-mrs.fr/IJFV/, vol. 4, 2007 , pp. 1-39.

3 Ambroso, A., Chalons, C., Coquel, F., Godlewski, E., Lagoutiere, F., Raviart, P.A. , And Seguin, N., "Relaxation methods and coupling procedures", International Journal for Numerical Methods in Fluids, vol. 56, 2008, pp. 1123-1129.

4 Ambroso, A., Chalons, C., Coquel, F., Godlewski, E., Lagoutiere, F., Raviart, P.A., And Seguin, N., "Coupling of general Lagrangian systems", Mathematics of Computation, vol. 77, 2008, pp. 909-941.

5 Ambroso, A., Hérard, J.M., And Hurisse, O., "A method to couple HEM and HRM two-phase flow models", Computers and Fluids, vol. 38, 2009, pp. 738-756.

6 Bachmann, F., "Equations hyperboliques scalaires à coefficients discontinus," Ph.D thesis, Aix-Marseille I University, Marseille, France, 2005.

7 Baer, M.R., And Nunziato, J.W., "A two-phase mixture theory for the deflagration to detonation transition (DDT) in reactive granular materials", Int. J. Multiphase Flow, vol. 12(6), 1986, pp. 861-889.

8 Bdzil, J.B., Menikoff, R., Son, S.F., Kapila, A.K., and Stewart, D.S., "Two-phase modeling of a DDT in granular materials: a critical examination of modeling issues", Phys. of Fluids, vol. 11, 1999, pp. 378-402.

9 Caetano, F., "Sur certains problèmes de linéarisation et de couplage pour les systèmes hyperboliques non-linéaires," Ph.D thesis, Pierre et Marie Curie University, Paris, France, December 12, 2007.

10 Chalons, C., " Theoretical and numerical aspects of the interfacial coupling: the scalar Riemann problem and an application to multiphase flow" Networks and Heterogeneous Media, vol.5(3), 2010, pp. 507-524.

11 Chalons, C., Raviart, P.A., And Seguin, N., "The interfacial coupling of the gas dynamics equations", Quart. Appl. Math., vol.66(4), 2008, pp. 659-705.

12 Coquel, F., Gallouët, T., Hérard, J.M., and Seguin, N., "Closure laws for a two-fluid two-pressure model", $C$. $R$. Acad. Sci. Paris, vol. I-332, 2002, pp. 927-932.

13 Galié, T., "Couplage interfacial de modèles en mécanique des fluides. Application aux écoulements diphasiques," Ph.D thesis, Pierre et Marie Curie University, Paris, France, March 31, 2009.

14 Gallouët, T., HÉrard, J.-M., and Seguin, N., "Numerical modelling of two phase flows using the two-fluid twopressure approach", Math. Mod. Meth. in Appl. Sci., vol. 14(5), 2004, pp. 663-700.

15 Girault, L., And Hérard, J.-M., "A two-fluid hyperbolic model in a porous medium", Math. Model. and Numer. Anal., vol. 44(6), 2010, pp. 1319-1348.

16 Giraul, T L., ANd HÉrard, J.-M., "Proposition pour un couplage interfacial instationnaire d'un modèle triphasique et d'un modèle diphasique", EDF report H-I81-2010-0355-FR, in French, 2010.

17 Girault, L., ANd HÉrard, J.M., "Multidimensional computations of a two-fluid hyperbolic model in a porous medium", AIAA paper 2009-3888, URL: http://www.aiaa.org/, 2009.

18 Godlewski, E. , "Coupling fluid models. Exploring some features of interfacial coupling", in the proceedings of "Finite Volumes for Complex Applications V", ISTE-Wiley, 2008, pp.87-102.

19 Godlewski, E., And Raviart, P.A., "The numerical interface coupling of non-linear hyperbolic systems of conservation laws. I The scalar case.", Numer. Math., vol. 97, 2004, pp. 81-130.

20 E. Godlewski, E., Le Thanh, K.C., and Raviart, P.A., "The numerical interface coupling of non-linear hyperbolic systems of conservation laws. II the case of systems", Mathematical Modelling and Numerical Analysis, vol.39, 2005, pp. 649-692.

21 Greenberg, J.M., And Leroux, A.Y., "A well-balanced scheme for the numerical processing of source terms in hyperbolic equations", SIAM Journal of Numerical Analysis, vol. 33, 1996, pp. 1-16.

22 Guelfi, A. , Bestion, D., Boucker, M. , Boudier, P., Fillion, P., Grandotto, M., Hérard, J.M. , Hervieu, E., AND PÉTURAud, P., "NEPTUNE: a new softaware platform for advanced nuclear thermal hydraulics", Nuclear Science and Engineering, vol. 156, 2007, pp. 281-324.

23 Helluy, P., Hérard, J.-M., And Mathis, H., "A well-balanced approximate Riemann solver for variable cross-section compressible flows", AIAA paper 2009-3540, URL: http://www.aiaa.org/, 2009.

24 Hérard, J.-M., "A rough scheme to couple free and porous media", International Journal of Finite Volumes, URL: http://www.latp.univ-mrs.fr/IJFV/, vol. 3(2), 2006, pp. 1-28.

25 HÉrard, J.-M., "A three-phase flow model", Mathematical Computer Modelling, vol. 45, 2007, pp. 432-455.

26 HÉrard, J.-M., "Un modèle hyperbolique diphasique bi-fluide en milieu poreux", Comptes-rendus Mécanique, vol. 336, 2008, pp. 650-655.

27 HÉrard, J.M. , AND Hurisse, O., "Couplage interfacial d'un modèle homogène et d'un modèle bifluide", EDF report H-I81-2006-04691-FR, in French, 2006.

28 Hérard, J.M. , ANd Hurisse, O., "Coupling two and one-dimensional unsteady Euler equations through a thin interface", Computers and Fluids, vol.36, 2007, pp. 651-666.

29 Hérard, J.M., ANd Hurisse, O., "Boundary conditions for the coupling of two-phase flow models", AIAA paper 20074458, URL: http://www.aiaa.org/, 2007.

30 Hérard, J.M., And Hurisse, O., "Some attempts to couple distinct fluid models", Networks and Heterogeneous Media, vol.5(3), 2010, pp. 649-660.

31 IshiI, M., "Thermofluid dynamic theory of two-phase flow", Collection de la Direction des Etudes et Recherches d'Electricité de France, Collection Eyrolles, 1975. 
32 Kapila, A.K., Son, S.F., Bdzil, J.B., Menikoff, R., And Stewart, D.S., "Two-phase modeling of a DDT: structure of the velocity relaxation zone", Phys. of Fluids, vol. 9(12), 1997, pp. 3885-3897.

33 Kröner, D., AND Thanh, M.D., "Numerical solutions to compressible flows in a nozzle with variable cross-section", SIAM Journal of Numerical Analysis, vol. 43, 2005, pp. 796-824.

34 Valette, M., And Jayanti, S., " Annular dispersed flow calculations with a two-phase three field model", European Two-Phase Flow Group Meeting, internal CEA report DTP/SMTH/LMDS/2003-085, 2003. 